\title{
La bronchiolite aiguë du nourrisson: à propos de 112 cas hospitalisés au département pédiatrie du CHU Gabriel Touré.
}

\section{Acute bronchiolitis of infants: about 112 cases hospitalized in the pediatric department of CHU Gabriel Touré}

Doumbia AK, Togo P, Coulibaly O, Dembélé A, Sacko K, Maiga B, Cissé ME, Konaté D, Traoré F, Diall H, Coulibaly A, Diakité AA, Dicko FT, Sylla M, Togo B.

Auteur correspondant : Dr Abdoul Karim Doumbia,Pédiatre CHU Gabriel Touré. Email : doumbiav@gmail.com

\section{RESUME}

Introduction : Les infections respiratoires aiguës (IRA) constituent l'une des principales causes de morbidité et de mortalité infantile au Mali. L'objectif de ce travail était de décrire les aspects épidémiologiques, cliniques et thérapeutiques de la bronchiolite aiguë du nourrisson au service de pédiatrie du CHU Gabriel Touré.

Méthodologie: Il s'agit d'une étude rétrospective portant sur 112 nourrissons âgés de 1 à 24 mois hospitalisés dans le service de pédiatrie générale pour bronchiolite aiguë. L'étude s'étalait sur une période d'un an (du $1^{\text {er }}$ janvier au 31 décembre 2012).

Résultats: Les nourrissons âgés de 1 à 6 mois étaient majoritaires (69\%). L'âge moyen des patients était de 6 mois avec des extrêmes de 1 et 24 mois. Le sexe masculin était prédominant $(63 \%)$ avec un sex-ratio de 1,73. Le principal motif de consultation était la difficulté respiratoire (87\%). Le pic d'hospitalisation était au mois de novembre (33\%). Les principaux signes cliniques en plus des sibilants étaient la toux, la rhinorrhée et la détresse respiratoire $(97 \%)$. La fièvre était présente dans $38 \%$ des cas et la cyanose chez $4 \%$ des patients. La saturation en oxygène était inférieure à $94 \% \mathrm{chez}$ $50 \%$ des patients. La kinésithérapie respiratoire a été faite chez un tiers des malades. Tous les malades avaient reçu une corticothérapie et une nébulisation avec du salbutamol et sérum physiologique. Une antibiothérapie a été faite chez $85 \%$ des patients. La durée moyenne d'hospitalisation était de 6 jours avec des extrêmes de 1 et 30 jours. Le taux de guérison était de $98 \%$.

Conclusion: La bronchiolite est une pathologie bénigne et fréquente chez le nourrisson de 1 à 6 mois qui évolue favorablement dans la majorité des cas. Mots-clés : Bronchiolite, infection, virus, nourrisson.

\section{ABSTRACT}

Introduction: Acutes Respiratory Infections (ARI) is one of the leading causes of morbidity and infant mortality in Mali. The objective of this work was to describe the epidemiological, clinical and therapeutic aspects of acute bronchiolitis in infants in the pediatric department of CHU Gabriel Touré. Methodology: This is a retrospective study of 112 infants aged 1 to 24 months hospitalized in the General Pediatric Department for acute bronchiolitis. The study was spread over a one-year period (January 1 to December 31, 2012).

Results: Infants aged 1 to 6 months were the majority $(69 \%)$. The average age of patients was 6 months with extremes of 1 and 24 months. The male sex was predominant $(63 \%)$ with a sex ratio of 1.73. The primary reason for consultation was difficulty breathing $(87 \%)$. The peak of hospitalization was in November $(33 \%)$. The main clinical signs in addition to the sibilants were cough, rhinorrhea and respiratory distress (97\%). Fever was present in $38 \%$ of cases and cyanosis in $4 \%$ of patients. Oxygen saturation was less than $94 \%$ in $50 \%$ of patients. Respiratory physiotherapy was done in one-third of the patients. All patients received corticosteroid therapy and nebulization with salbutamol and saline. Antibiotic therapy was performed in $85 \%$ of patients. The average duration of hospitalization was 6 days with extremes of 1 and 30 days. The cure rate was $98 \%$.

Conclusion: Bronchiolitis is a benign and frequent pathology in the infants from 1 to 6 months, which evolves favorably in the majority of the cases. Keywords: Bronchiolitis, infection, virus, infant

\section{INTRODUCTION}

La bronchiolite aiguë du nourrisson est une infection virale aiguë caractérisée par une dyspnée à prédominance expiratoire. Elle peut être associée à une polypnée, des signes de lutte respiratoires, un wheezing, des sibilants ou des crépitants $[1,2,6]$. Dans les formes les plus graves, l'auscultation peut être silencieuse chez un nourrisson sur-distendu [3]. Plusieurs virus sont responsables d'une bronchiolite aiguë mais le virus respiratoire syncitial (VRS) est de loin le plus représenté [2]. La bronchiolite affecte généralement les enfants de moins de deux ans, avec un pic d'incidence entre deux et six mois [16]. Au Royaume-Uni, environ 1 nourrisson sur 3 développe une bronchiolite dans la première année de vie et 2 à $3 \%$ d'entre eux seront hospitalisés [5]. L'importance de la bronchiolite n'est pas évaluée au Mali avec l'absence de système de surveillance nationale. Les décès liés à cette maladie sont rares [2]. L'objectif de notre travail était d'étudier les aspects épidémiologiques, cliniques et thérapeutiques de la bronchiolite aiguë du nourrisson au service de pédiatrie du CHU Gabriel Touré.

\section{MATÉRIEL ET MÉTHODES}


Il s'agissait d'une étude rétrospective et descriptive à partir des dossiers médicaux des nourrissons âgés de 1 mois à 24 mois, hospitalisés pour bronchiolite. Elle s'est étalée sur une période de 12 mois allant du $1^{\text {er }}$ janvier au 31 Décembre 2012. Ce travail s'est déroulé au service de pédiatrie générale du CHU Gabriel Touré qui est un hôpital de $3^{\text {ème }}$ référence au sommet de la pyramide sanitaire du Mali. Nous avons inclus tous les nourrissons atteints de bronchiolites. Le questionnaire d'enquête comportait les variables suivants :

- les caractéristiques socio-démographiques (résidence, sexe, âge, ethnie),

- les signes cliniques (état général, signes de lutte respiratoire, température, fréquence respiratoire, saturation, anomalies auscultatoires, signes associés),

- les données para cliniques (NFS, radiographie pulmonaire, saturation)

- les aspects thérapeutiques et évolutifs.

La saisie des données a été faite avec le logiciel Epi info version $6.4 \mathrm{~d}$ fr. Le traitement de texte a été fait sur Microsoft Word 2010.

\section{RESULTATS}

1- Données épidémiologiques

Durant la période d'étude, 10605 enfants ont été hospitalisés dans le département de pédiatrie du CHU Gabriel Touré. Nous avons enregistré 1238 cas d'infections respiratoires basses parmi lesquels 112 patients atteints de bronchiolite. La bronchiolite représentait $1,05 \%$ du total des admissions en pédiatrie et $9,04 \%$ des hospitalisations pour infection respiratoire aiguë basse (IRAB).Les garçons représentaient $63 \%$ de l'échantillon et les filles $37 \%$ soit un sex-ratio de 1,73. L'âge moyen des patients était de 6 mois avec des extrêmes de 1 et 24 mois. Les nourrissons de 1 à 6 mois représentaient $69 \%$ de l'échantillon, $17 \%$ avaient entre 7 et 12 mois et $14 \%$ avaient entre 13 à 24 mois. Le pic de fréquence (Figure 1) était obtenu au mois de novembre (33\%). La notion d'asthme familial a été retrouvée chez $16 \%$ des patients (Tableau I). Les vaccinations recommandées par le programme élargi de vaccination n'étaient pas correctement réalisées chez 6,3\%. Les patients étaient admis entre 18 heures et 08 heures du matin dans $56 \%$ des cas.

\section{2- Données cliniques et paracliniques}

Le principal motif de consultation était la gêne respiratoire (dyspnée) avec 87\% (Tableau II). Le délai de consultation était inférieur à cinq jours chez $72 \%$ des patients. Les principaux signes associés aux sibilants étaient la détresse respiratoire (96\%), la toux $(80 \%)$ et la rhinorrhée $(80 \%)$. La fièvre était présente chez $38 \%$ des patients, la déshydratation chez $7 \%$ et la cyanose chez $4 \%$ (Tableau III). La radiographie pulmonaire de face a été faite chez 71,42\% des patients. Elle avait monté une distension thoracique chez $71 \%$ des patients et des opacités dans $29 \%$ des cas. L'hémogramme a été réalisé chez $51 \%$ des patients. La saturation était inférieure à $94 \%$ chez $50 \%$ des patients.

\section{3- Données thérapeutiques et évolutives}

Les mesures générales telles que la désobstruction rhinopharyngée (DRP) avec du sérum physiologique, la mise en position proclive à $30^{\circ}$ avec la tête en légère extension, le maintien d'une hydratation correcte, la nutrition par gavage gastrique discontinu en cas de fausses routes ou de difficultés d'alimentation, étaient systématiques. L'oxygénothérapie par lunette nasale a été indiquée chez les patients qui présentaient une saturation en oxygène à l'air ambiant inférieure à 94\% (50\% des patients). La kinésithérapie respiratoire a été faite chez $30 \%$ de nos malades. Tous les patients ont reçu une corticothérapie. La durée de cette corticothérapie systémique variait entre 1 et 6 jours. La nébulisation avec le salbutamol associé au sérum physiologique a été également réalisée chez tous les patients. L'antibiothérapie a été faite chez $85 \%$ des patients. La durée moyenne d'hospitalisation était de 5,8 jours avec des extrêmes allant de 1 et 30 jours. L'évolution était favorable chez 98,2\% des patients. La létalité était de 1,8\%.

\section{DISCUSSION.}

1- Discussion sur la méthode :

Cette étude rétrospective présente quelques insuffisances dues à la méthodologie utilisée. Certaines données anamnestiques et cliniques ne figurent pas dans tous les dossiers, notamment la présence ou non d'un tabagisme parental ou les informations néonatales, les modalités de certains traitements.

2- Epidémiologie L'incidence de la bronchiolite est mal connue au Mali. Sa fréquence est élevée en milieu hospitalier $(1,05 \%)$. Nos résultats sont très proches de ceux obtenus par Bogne et al., au Cameroun, où la bronchiolite représenterait $9,4 \%$ des infections respiratoires aigües basses et $1,6 \%$ des consultations pédiatriques [17]. Ce taux hospitalier peut s'expliquer par une absence de protocole définissant les critères de gravité ou d'hospitalisation dans le service. Par ailleurs nos chiffres étaient inférieurs à celles de Bobossi et al., à Bangui $(20,9 \%$ des IRAB et $1,7 \%$ des hospitalisations) [13]. La bronchiolite représente 2 à $3 \%$ des hospitalisations chez les nourrissons âgés de moins d'1 an au Royaume-Uni [6]. La prédominance masculine dans cette affection a été rapportée par plusieurs auteurs [4, 5, 13]. Selon l'Institut de Veille Sanitaire en France, dans $59 \%$ des cas, les nourrissons hospitalisés pour bronchiolite en 2012, étaient des garçons. Malgré ces constats, nous estimons que le sexe ne semble jouer aucun rôle dans la survenue de la bronchiolite. La bronchiolite aiguë est plus fréquente durant la première année de vie. La majorité de nos patients avait moins de 6 mois (67\%) et $86 \%$ avaient moins d'un an. Nos résultats sont concordants avec ceux 
de Bogne et al., et de Bobossi et al., à Bangui $[4,13]$. Les mêmes tendances sont confirmées dans une étude prospective réalisée en milieu hospitalier en Inde du Sud où sur 114 enfants atteints de bronchiolite, 87 patients (soit $76 \%$ ) avaient moins de 1 an et 107 (soit 94\% des patients) avaient moins de 2 ans [16]. La majorité des patients $(72,3 \%)$ avait consulté dans les cinq jours après le début des symptômes. Malgré ce délai jugé court par rapport à l'étude de Bogne au Cameroun (7 jours), la plupart était admis dans un tableau de détresse respiratoire sévère d'où leur hospitalisation. La majorité des patients $(66,07 \%)$ était hospitalisée entre septembre et décembre, à la fin de la saison des pluies et au début de la saison sèche. Le pic d'hospitalisation était obtenu au mois de novembre en tout début de la saison sèche (33\%). Le plus faible nombre de cas était observé aux mois de février et Mai, période de forte chaleur où les températures dépassent les 40 degrés. Nos résultats concordent avec ceux de Bobossi et al., qui ont recruté plus de patients durant la saison sèche où les mois sont frais [13]. Au Cameroun, la bronchiolite aiguë du nourrisson serait plus fréquente pendant la grande saison des pluies, de septembre à novembre avec un pic en octobre [17]. Dans les pays du nord nous retrouvons une augmentation de la fréquence pendant la période hivernale $[4,6]$.

3- Clinique

A l'admission, la symptomatologie clinique dominante en dehors des sibilants était la difficulté respiratoire. Dans l'étude de Zariat Makrem, la majorité $(70 \%)$ avait des signes de lutte respiratoire [4]. La fréquence élevée de ce symptôme pourrait s'expliquer par la pathogénèse de la maladie. Dans la population de nourrissons étudiée par Gueddari et al., au Maroc, le premier motif de consultation était la fièvre [11]. La fièvre est un signe qui inquiète les parents mais elle n'est pas forcément un critère de gravité. Cependant la fièvre peut signifier une co-infection ou une surinfection et justifier alors un dosage de CRP, une NFS, une radiographie pulmonaire [8]. La surinfection bactérienne des voies respiratoires au cours des bronchiolites reste difficile à évaluer [6]. Plusieurs études ont montré des résultats hétérogènes. Malgré l'absence de consensus sur les critères d'hospitalisation dans le service, nous pensons que les décisions d'hospitalisation étaient cliniquement justifiées chez la plupart des patients. Dans la littérature ces critères reposent sur le jugement clinique et tiennent compte de l'état respiratoire du nourrisson, de son état d'hydratation, du risque d'évolution vers une maladie grave et de la capacité qu'a la famille d'affronter la situation [8].La mesure de la saturation en oxygène permet d'apprécier la gravité de la bronchiolite. Pour certains auteurs, son rôle demeure controversé pour prendre des décisions cliniques $[8,14]$.
La radiographie pulmonaire était assez prescrite ( $71,4 \%$ des enfants) alors que son indication devait se limitée au seul cas où la gravité de l'évolution laisse supposer un autre diagnostic $[4,8]$. Le nombre de radiographies pulmonaires effectuées dépassait largement le nombre d'enfant ayant de la fièvre $(38 \%)$ ou des râles crépitants $(58 \%)$. Alors que la radiographie thoracique n'a pas d'intérêt au cours de la bronchiolite typique sans signe de gravité $[5,8]$. La bronchiolite étant une infection virale, la recherche systématique de signe d'infection bactérienne comme une hyperleucocytose à polynucléaires neutrophiles et le dosage de la CRP n'a pas d'intérêt sauf en cas de surinfection suspectée $[4,14]$. Les tests sérologiques et les prélèvements des secrétions bronchiques n'ont pas été faits. Ces analyses sont nécessaires seulement pour établir la cohorte des patients hospitalisés [8].

\section{4- Aspects thérapeutiques}

La prise en charge d'une bronchiolite du nourrisson est essentiellement symptomatique [1, 2]. Les recommandations thérapeutiques sont généralement le fruit de l'expérience quotidienne. Dans la majorité des cas, la bronchiolite ne nécessite pas une hospitalisation [2, 8]. Le désencombrement rhinopharyngé par des instillations nasales avec du sérum physiologique narine par narine a été fait chez tous les patients. Il était souvent complété par une aspiration mécanique par sonde d'aspiration chez les nourrissons les plus symptomatiques. Une hydratation par voie parentérale était faite chez 7\% des patients présentant des formes sévères de bronchiolite avec dyspnée. Dans notre série, la kinésithérapie au cours de la phase obstructive et hypersécrétante de la maladie avait été faite. Plusieurs auteurs surtout anglo-saxons pensent que les techniques de kinésithérapie respiratoire ne réduisent pas la durée du séjour hospitalier et n'améliorent pas les scores de gravité clinique chez le nourrisson atteint de bronchiolite $[1,2,8,9]$. En France, la prescription de la kinésithérapie dans la bronchiolite varie de $82.5 \%$ à $99 \%$ [2, 18]. Les corticoïdes et les bronchodilatateurs (salbutamol) sont largement prescrits en milieu hospitalier pédiatrique. Tous les nourrissons inclus à l'étude avaient reçu une corticothérapie. De nombreuses études témoignent de l'absence d'intérêt des corticoïdes et du faible bénéfice des bronchodilatateurs $[2,8,9,10]$. Cette absence d'efficacité des bronchodilatateurs serait probablement en rapport avec la physiopathologie de la bronchiolite qui est différente de l'asthme. En effet, l'obstruction de la bronchiolite est mécanique et le bronchospasme ne joue qu'un rôle mineur contrairement à l'asthme [8, 12]. Dans la littérature, la prise en charge hospitalière de la bronchiolite ne varie pas assez d'un auteur à l'autre : 99\% des pédiatres suisses utilisent le salbutamol, $80 \%$ des pédiatres canadiens prescrivent un 
bronchodilatateur pour traiter la bronchiolite [2, 8 , 14]. Des études françaises ont montré que les bronchodilatateurs apparaissaient encore dans 40 à $60 \%$ des prescriptions lors d'une bronchiolite contrairement aux recommandations [1, 2, 18]. Plusieurs études montrent l'absence de bénéfice des antibiotiques dans la bronchiolite [5, 7, 8]. Chez G. Bobossi-Serengbe et al., tous les patients avaient bénéficié d'une antibiothérapie.

5- Aspects évolutifs

Avec une durée moyenne de séjour de 6 jours, nos résultats sont statistiquement peu alarmants. Dans l'étude de Bobossi-serengbe et al., le délai moyen d'hospitalisation était de 8,3 jours. En Inde, chez les nourrissons auparavant en bonne santé, la durée moyenne d'hospitalisation est de trois à quatre jours [16]. Le séjour peut être prolongée chez les enfants plus jeunes et ceux qui ont des conditions de comorbidité (par exemple, maladie pulmonaire chronique) $[6,16]$. L'évolution était généralement favorable chez nos malades $(98 \%)$ comme dans l'étude de Ghedira et al., (98,6\%). La bronchiolite est une maladie avec une morbidité élevée, mais une faible mortalité [5]. Nous avons recensé deux cas de décès âgés respectivement de 3 et 8 mois. Le premier était un enfant trisomique arrivé aux urgences vers 10 heures du matin dans un tableau d'hyperthermie $\left(39^{\circ} 5\right)$, de détresse respiratoire sévère et une désaturation à $77 \%$. Le second avait 8 mois, il était arrivé très épuisé, fébrile et en hypoxie vers 22 heures. Ils étaient tous les deux à leur première crise. Chez Bobossi-serengbe et al., la létalité était de $20 \%$ [13]. Cette forte létalité était surtout due au recours tardif de soins et au manque de moyens efficaces de réanimation. Dans l'étude de Bogne, le pronostic global était favorable dans $98,9 \%$ des cas avec une létalité de 1,1\% [17].

\section{CONCLUSION}

La bronchiolite constitue un véritable problème de santé publique. Elle affecte généralement les enfants de moins de deux ans, avec un pic d'incidence entre deux et six mois. La fréquence des hospitalisations est plus élevée au mois de Novembre (33 \%). Son diagnostic est essentiellement clinique et les examens de laboratoire ont un rôle limité dans le diagnostic et la gestion. Le traitement de la bronchiolite est essentiellement symptomatique et vise au maintien d'une oxygénation et d'une hydratation adéquate. L'évolution clinique des patients est généralement favorable mais la létalité hospitalière au Mali n'est pas négligeable $(1,8 \%)$. L'amélioration de la prise en charge hospitalière des nourrissons atteints de bronchiolite passera par la formation des agents de santé à la prise en charge des cas, la promotion de projets de recherche médicale et l'élaboration de protocole consensuel de prise en charge.

\section{- REFERENCES}

1. Ernst Eber. Treatment of Acute Viral Bronchiolitis. The Open Microbiology Journal, 2011, 5, (Suppl 2-M6) 159-164.

2. Barben J, Hammer J. Current management of acute bronchiolitis in Switzerland. Swiss Med Wkly 2003; 133: 9-15.

3. Freymuth F. Human respiratory syncytial virus and Para-influenza virus: epidemiology. EMCPédiatrie 1 (2004) 2-11.

4. Zariat M. Evaluation de la pertinence d'hospitalisation des enfants atteints de bronchiolite dans un service de pédiatrie générale en île de France pour les saisons automne -hiver 2004-2005 et 2005-2006. [Thèse de médecine]. Université Paris Val-De-Marne Faculté de médecine de Créteil, 2009. Paris-France : 26 - 29.

5. Øymar K, Skjerven HO, Mikalsen IB. Acute bronchiolitis in infants, a review. Scand J Trauma Resusc Emerg Med. 2014; 22: 23.

6. Andrew Bush, Anne $\mathbf{H}$ Thomson. Acute bronchiolitis. BMJ 2007; 335:1037-41.

7. Claudia Ravaglia et Venerino Poletti. Recent advances in the management of acute bronchiolitis. F1000Prime Reports 2014, 6:103 (doi: 10.12703/P6-103)

8. Jeremy $\mathbf{N}$ Friedman, Rieder MJ, et al. Bronchiolitis: Recommendations for diagnosis, monitoring and management of children one to 24 months of age. Paediatr Child Health Vol 19 No 9 November 2014; 485 - 491.

9. Didier Evenou, Pelca D. « Bronchiolite Ile-deFrance ». Revue de presse. Soins pédiatriepuériculture $\mathrm{n}^{\circ} 202$ octobre 2001 .

10. Brouard. J. Bronchiolite à VRS : clinique et prise en charge de la bronchiolite à VRS. Urgence pratique. 2005; $\mathrm{n}^{\circ} 70: 11,12,13$.

11. Gueddari W, Tazi A, Ouardi A, Nani S et al. Evaluation of parental knowledge on acute bronchiolitis. The Pan African Medical Journal. 2014; 17: 310

12. Dutau G, Labbé A. Bronchiolite à VRS : une infection pédiatrique d'actualité. Paris. Doin Edit ; 2005 : pages 7 - 10.

13. Bobossi-Serengbe G, Bangue C, Mobima T. Les aspects épidémiologiques, cliniques et thérapeutiques des bronchiolites aiguës du nourrisson au complexe pédiatrique de Bangui (Centrafrique) Méd Afr Noire - 2004 ; 217-222.

14. Unger S, Cunningham S. Effect of oxygen supplementation on length of stay for infants hospitalized with acute viral bronchiolitis. Pediatrics 2008; 121: 470-5.

15. INVS. Epidémie de bronchiolite à la Réunion. Point épidémiologique - $\mathrm{N}^{\circ} 87$ du 27 décembre 2012. Page 1-3.

16. Nishant Verma, Rakesh Lodha, S K Kabra. Recent Advances in Management of Bronchiolitis. Indian Pediatr 2013;50: 939-949.

17. Bogne JB, Chiabi A, Yatchet $D$ et al. Bronchiolite aiguë du nourrisson de moins de 24 
mois à Yaoundé (à propos de 296 cas).Health.Sci.Dis Vol 14, No4 (2013).

Tableau I : Répartition des patients selon les antécédents

\begin{tabular}{|l|c|c|}
\hline Antécédents & Effectif & Pourcentage \\
\hline Faible Poids de Naissance & 5 & $\mathbf{4 , 5}$ \\
\hline Détresse Respiratoire du Nouveau-né & 4 & $\mathbf{3 , 6}$ \\
\hline Atopie familiale & 15 & $\mathbf{1 3 , 4}$ \\
\hline Prématurité & 1 & $\mathbf{0 , 9}$ \\
\hline Cardiopathie congénitale & 6 & $\mathbf{5 , 4}$ \\
\hline \hline Notion d'asthme familial & $\mathbf{1 8}$ & $\mathbf{1 6 , 1}$ \\
\hline
\end{tabular}

Les comorbidités étaient fréquentes avec $\mathbf{2 7 , 6 \%}$ (31cas) des malades inclus dans notre étude. La notion d'asthme familial a été retrouvée chez $\mathbf{1 6 , 1 \%}$ des patients.

Tableau II : Répartition des patients selon le motif de consultation (plaintes)

\begin{tabular}{|l|c|c|}
\hline Motif de consultation & Effectif & Pourcentage \\
\hline Difficultés respiratoires & 97 & $\mathbf{8 6 , 6}$ \\
\hline Toux & 33 & $\mathbf{2 9 , 5}$ \\
\hline Fièvre & 26 & $\mathbf{2 3 , 2}$ \\
\hline Douleur thoracique & 2 & $\mathbf{1 , 8}$ \\
\hline \hline Autres * & $\mathbf{4}$ & $\mathbf{3 , 6}$ \\
\hline
\end{tabular}

Autres* : vomissement $=1$, diarrhée $=1$, jaunisse $=1$, rhinorrhée $=1$

Le motif de consultation le plus fréquent était les difficultés respiratoires avec $86,6 \%$.

Tableau III: Caractéristiques cliniques

\begin{tabular}{|l|c|c|}
\hline Signes cliniques & Effectif & Pourcentage \\
\hline Température $\geq \mathbf{3 8}^{\circ} \mathbf{C}$ & 43 & $\mathbf{3 8}$ \\
\hline Cyanose & 4 & $\mathbf{3 , 6}$ \\
\hline Signes de lutte & 107 & $\mathbf{9 5 , 5}$ \\
\hline Polypnée & 73 & $\mathbf{6 5 , 2}$ \\
\hline Râles crépitants & 65 & $\mathbf{5 8}$ \\
\hline Rhinorrhée & 109 & $\mathbf{9 7}$ \\
\hline Déshydratation & 8 & $\mathbf{7 , 1}$ \\
\hline Vomissement & 11 & $\mathbf{9 , 8}$ \\
\hline Refus d'alimentation & 33 & $\mathbf{2 9 , 5}$ \\
\hline Fausse route & 2 & $\mathbf{1 , 8}$ \\
\hline \hline Convulsion & $\mathbf{1}$ & $\mathbf{0 , 9}$ \\
\hline
\end{tabular}

Les signes de lutte étaient plus fréquemment représentés $(95,5 \%)$. 


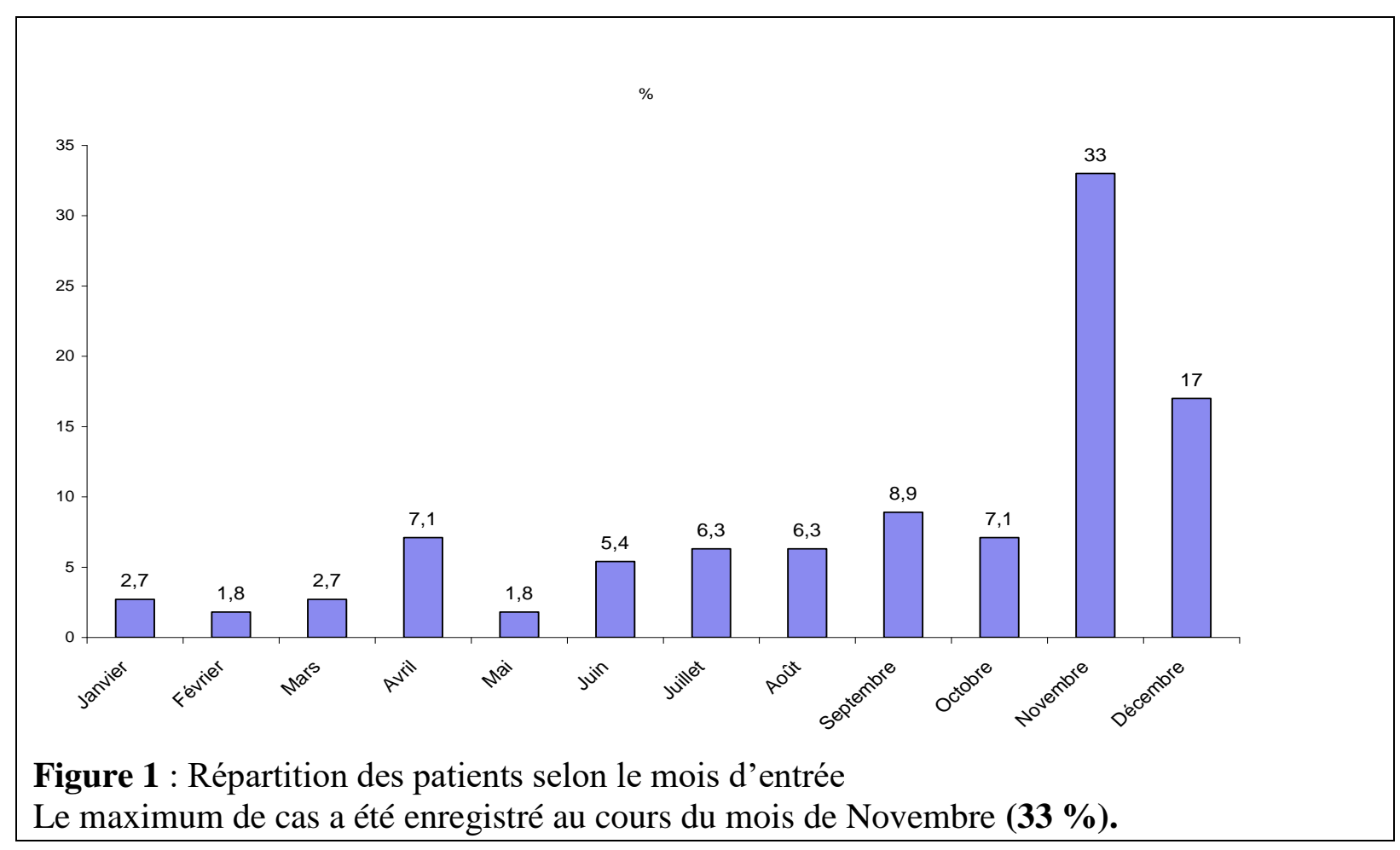

